\title{
Publisher's Note: Stochastic models for objects and images in oncology and virology: application to PI3K-Akt-mTOR signaling and COVID-19 disease
}

\author{
Harrison H. Barrett ${ }^{\mathrm{a}, \mathrm{b}}$ and Luca Caucci ${ }^{\mathrm{a}, \mathrm{b}}$ \\ ${ }^{a}$ University of Arizona, Wyant College of Optical Sciences, Tucson, Arizona, United States \\ ${ }^{b}$ University of Arizona, Department of Medical Imaging, Arizona, United States
}

This article [J. Med. Imag. 8(S1), S16001 (2020) doi: 10.1117/1.JMI.8.S1.S16001 was originally published in Vol. 8, No. S1 of the Journal of Medical Imaging (JMI) on 1 December 2020 with an incorrect digital object identifier (DOI) of 10.1117/1.JMI.7.6.S16001. The DOI was corrected and registered as 10.1117/1.JMI.8.S1.S16001 on 3 December 2020.

The typical structure of the DOI for JMI uses the first seven digits to identify the publisher, and the final 8 digits to indicate the volume number, issue, and citation identifier (CID). The corrected DOI indicates that the article was published in Volume 8, Special Issue 1. 